\title{
Theory of Inverse Demand: Financial Assets
}

\author{
Felix Kubler \\ University of Zurich \\ Swiss Finance Institute
}

\author{
Larry Selden \\ Columbia University \\ University of Pennsylvania
}

\author{
Xiao Wei \\ Columbia University
}

April 2, 2011

\begin{abstract}
While the comparative statics of asset demand have been studied extensively, surprisingly little work has been done on the behavior of equilibrium asset prices and returns in response to changes in the supplies of securities. This is despite considerable interest in the equity premium and interest rate puzzles. In this paper, we seek to fill this void for the classic case of a representative agent economy with a single risky asset and risk free asset in both one and two period settings. It would seem natural to suppose that in response to an increase in the supply of the risky asset, its price would fall and the gross equity risk premium would increase. We show that in standard settings where preferences are represented by frequently assumed forms of expected utility, one can obtain the opposite result. The necessary and sufficient condition for prices (gross equity premium) to increase (decrease) with supply is determined by the sign of the slope of the asset Engel curve. This observation allows us to derive (i) sufficient conditions directly in terms of the representative agent's risk aversion properties for general utility functions and (ii) necessary and sufficient conditions for the widely used HARA (hyperbolic absolute risk aversion) class.
\end{abstract}




\section{Introduction}

In this paper, we examine the behavior of equilibrium asset prices and returns in response to changes in the supplies of securities for the classic case of a single risky asset and risk free asset in both one and two period settings. We show that in standard single period and two period representative agent exchange economies where preferences are represented by frequently assumed forms of utility, it might very well be the case that an increase in the supply of the risky asset leads to a decrease of the gross equity premium.

Assuming the representative agent's preferences satisfy the appropriate expected utility axioms, we show that in a single period exchange economy there is a close linkage between the demand and equilibrium price comparative statics. The only related work of which we are aware, addresses the relationship between demand and equilibrium price comparative statics in a certainty framework. Nachbar [19] shows that a necessary condition for the equilibrium price of a good to increase with supply is the normal-inferior good behavior of demand (see also Nachbar [19] and Quah [24]). ${ }^{1}$ It is natural to wonder if similar results hold in the uncertain financial asset setting, given the recent findings of Kubler, Selden and Wei [14], that the risk free asset can be an inferior good if either short selling of the risk free asset is allowed or relative risk aversion is decreasing.

We identify the necessary and sufficient conditions for when asset prices (gross equity premium) increase (decreases) with asset supplies. The single period case turns out to be quite special in that there is a full equivalence between the comparative statics of demand and the equilibrium price ratio. The necessary and sufficient condition for prices (gross equity premium) to increase (decrease) with supply is determined by the sign of the slope of the asset Engel curve. This observation allows us to derive (i) sufficient conditions directly in terms of the representative agent's risk aversion properties for general utility functions and (ii) necessary and sufficient conditions for the widely used HARA (hyperbolic absolute risk aversion) class. $^{2}$

We extend our single period analysis to two periods. Once period one consumption is introduced as a third good, the equivalence between the demand and equilibrium price comparative statics breaks down. While the demand comparative statics become significantly more complex, most of the single period equilibrium comparative statics results based on the representative agent's general and HARA preferences extend from the single period setting. Moreover by selecting period one consumption as the numeraire, one can derive simple sufficient conditions for the comparative statics of the equilibrium expected return and the risk free rate.

In Section 2, we derive single period comparative statics corresponding to changes in asset supplies. Section 3 extends our analysis to two periods and considers an alternative specification for preferences. The final Section contains concluding comments.

\section{Single Period Comparative Statics}

In this Section, we consider a single period setting in which the representative agent with a given endowment of assets selects asset demands so as to maximize expected utility for end of period consumption. In the next Section, we consider the natural extension to a two period setting where the representative agent at the beginning of period one chooses both the level of certain consumption

\footnotetext{
${ }^{1}$ In a distribution economy setting, see Malinvaud's classic ([16], chapter 5) and the later contribution of Kohli [13].

${ }^{2}$ See [8], pp. 26-27, for a discussion of the HARA class and the properties of its members.
} 
$c_{1}$ as well as asset holdings the returns on which fund period two consumption, $c_{2}$. The notational conventions and preference structure of the current Section facilitate a simple transition to the two period problem in Section 3.

\subsection{Economy}

Assume the classic risky asset and risk free asset setting. Let the risky asset have random payoff $\widetilde{\xi}>0$ and a corresponding arbitrary cumulative distribution function $F(\widetilde{\xi})$. There also exists a risk-free asset with payoff $\xi_{f}>0$. Let $n$ and $n_{f}$ denote the number of units of the risky asset and risk free asset, respectively. The prices of the risky and risk free assets are given by $p$ and $p_{f}$, respectively. In the current single period setting, the representative agent's preferences are defined over random $\widetilde{c_{2}}=\widetilde{\xi} n+\xi_{f} n_{f}>k$, where $k$ is 0 or some positive constant, and satisfy the standard expected utility axioms where the NM (von Neumann-Morgenstern) index $W\left(c_{2}\right)$ satisfies $W \in C^{3}$, $W^{\prime}>0$ and $W^{\prime \prime}<0 .{ }^{3}$ The expected utility function $E W\left(\widetilde{c}_{2}\right)$ given by

$$
W\left(n, n_{f}\right)=E W\left(\widetilde{\xi} n+\xi_{f} n_{f}\right)=\int W\left(\widetilde{\xi} n+\xi_{f} n_{f}\right) d F(\widetilde{\xi}) .
$$

Then as is well-known, $W$ will be strictly increasing and concave in both $n$ and $n_{f}$.

The representative agent can be viewed as solving the optimization problem

$$
\max _{n, n_{f}} W\left(n, n_{f}\right)=\max _{n, n_{f}} E W\left(\widetilde{\xi}_{n}+\xi_{f} n_{f}\right)
$$

subject to

$$
p n+p_{f} n_{f}=p \bar{n}+p_{f} \bar{n}_{f},
$$

where $\bar{n}$ and $\bar{n}_{f}$ denote, respectively, endowments of the risky and risk free assets. The resulting first order condition is given by ${ }^{4}$

$$
\frac{W_{n}}{W_{n_{f}}}=\frac{E\left[\widetilde{\xi} W^{\prime}\left(\widetilde{\xi} n+\xi_{f} n_{f}\right)\right]}{E\left[\xi_{f} W^{\prime}\left(\widetilde{\xi} n+\xi_{f} n_{f}\right)\right]}=\frac{p}{p_{f}} .
$$

In this representative agent setting, the no bankruptcy condition is given by

$$
\bar{n} \inf \widetilde{\xi}+\xi_{f} \bar{n}_{f}>k .
$$

Throughout this paper, we also assume that

$$
\frac{E \widetilde{\xi}}{p}>\frac{\xi_{f}}{p_{f}}
$$

from which it follows that $n>0 .{ }^{5}$ As a result we assume a positive endowment of the risky asset $\bar{n}>0$.

\footnotetext{
${ }^{3}$ These single period NM preferences are extended in Section 3 to the two period expected utility $E W\left(c_{1}, \widetilde{c}_{2}\right)=$ $W_{1}\left(c_{1}\right)+E W_{2}\left(\widetilde{c}_{2}\right)$, where the consumer is choosing over both $c_{1}$ and $\left(n, n_{f}\right)$. The single period NM utility considered here can be viewed as corresponding to $W_{2}\left(\widetilde{c}_{2}\right)$ in the two period $E W\left(c_{1}, \widetilde{c}_{2}\right)$.

${ }^{4}$ Throughout this paper we define $W_{n}=\frac{\partial E W\left(\widetilde{\xi} n+\xi_{f} n_{f}\right)}{\partial n}, W_{n, n}=\frac{\partial^{2} E W\left(\widetilde{\xi} n+\xi_{f} n_{f}\right)}{\partial n^{2}}$. Other terms such as $W_{n_{f}}, W_{n, n_{f}}$ and $W_{n_{f}, n_{f}}$ are defined similarly.

${ }^{5}$ To see that $n>0$, note first that from eqn. (4)

$$
E\left[\left(\widetilde{\xi}-\frac{p}{p_{f}} \xi_{f}\right) W^{\prime}\left(\widetilde{\xi} n+\xi_{f} n_{f}\right)\right]=0 .
$$
}


On the other hand, we allow $\bar{n}_{f} \gtreqless 0$, which runs contrary to the conventional assumption that the supply of bonds is zero (e.g., [3]). In recent years, a number of papers have appeared which consider the case of a positive net supply of bonds. Heaton and Lucas [10] consider the existence of an outside supply of bonds, treating bonds as a Lucas [15] "tree" technology with a dividend equal to the equilibrium interest rate. Cochrane, Longstaff and Santa-Clara [5] generalize the Lucas tree structure employing a two tree model and briefly consider one tree being a bond. Parlour, Stanton and Walden [22] require a positive supply of bonds in order to make progress on resolving the equity premium, risk free rate and excess volatility puzzles. They state

The assumption that bonds are in zero net supply is consistent with an infinitely lived representative agent in an economy absent any frictions...By contrast, in a world with finitely lived investors, or with frictions, it may be possible for the current generation to borrow against the consumption of future generations, leading to a positive supply of bonds and risk-free consumption for the current generation over a significant time period. Indeed, in any economy in which Ricardian equivalence fails, government bonds can be in positive net supply. ([22], p. 3)

While the above references assume a positive supply of bonds, Favilukis, Ludvigson and Van Nieuwerburgh [7] assume a negative supply of bonds which they motivate by foreigners holding US debt. Thus, in the case where $\bar{n}_{f}>0$, the issuer of the bonds (for example, the Government) is outside the model. Analogously if $\bar{n}_{f}<0$, it is the lender that is outside the model.

Finally, Cass and Pavlova [4] observe that while nonnegativity assumptions for commodity endowments are very defensible, there is nothing contradictory in dropping this assumption when considering financial assets, especially when there are no restrictions on asset trade. In this paper, we drop the zero net supply assumption for the risk free asset. By not restricting $\bar{n}_{f}=0$, we will be able to derive a number of interesting differences in the comparative statics of equilibrium returns and gross equity premium corresponding to changes in asset supplies.

Given the representative agent setting specified above, it is clear that there will be a unique equilibrium defined by $\left(p, p_{f}, n, n_{f}\right)$. This equilibrium corresponds to the fixed parameter set $\left(\bar{n}, \bar{n}_{f}, \widetilde{\xi}, \xi_{f}\right)$ where equilibrium prices are endogenous.

\subsection{Inverse Demand and Equity Premium Behavior}

Following Katzner [11] when solving the representative agent's demand problem eqns. (2)-(3), one can think of fixing the budget constraint based on a given endowment and prices and finding the maximum utility value. On the other hand, when solving for equilibrium prices, one fixes the specific indifference curve passing through the endowment point and then solves for the equilibrium price ratio equal to the slope of the tangent to the indifference curve at that point. In both instances, the optimal point corresponds to the tangent point $\left(\bar{n}, \bar{n}_{f}\right)$ in Figure 1.

Clearly, we have

$$
E\left[\left(\widetilde{\xi}-\frac{p}{p_{f}} \xi_{f}\right) W^{\prime}\right] \lesseqgtr\left(E \widetilde{\xi}-\frac{p}{p_{f}} \xi_{f}\right) E W^{\prime} \Leftrightarrow n \gtreqless 0 .
$$

Therefore, our assumption that

$$
\frac{E \widetilde{\xi}}{p}>\frac{\xi_{f}}{p_{f}}
$$

implies that $n>0$. This argument continues to hold in the two period case considered in Section 3 since the first order condition for $n$ and $n_{f}$ remains the same. 


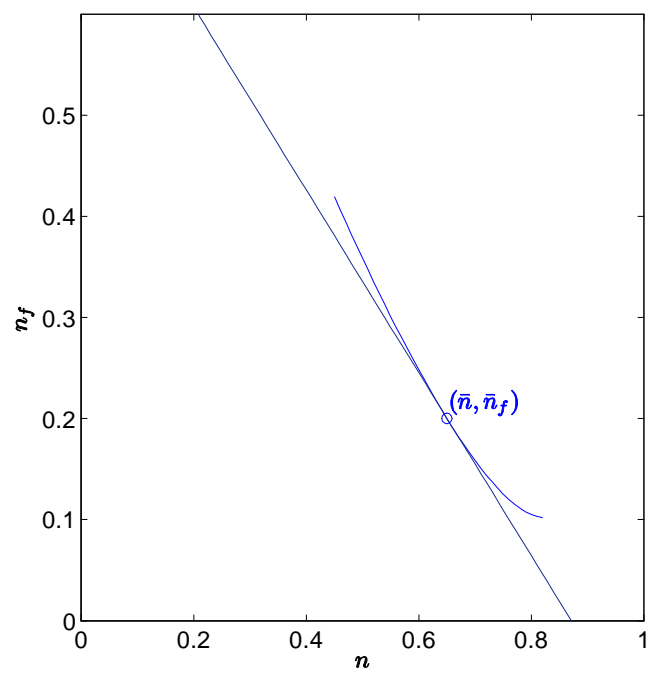

Figure 1:

Next we argue that in the single period case, the necessary and sufficient conditions for signing the equilibrium price ratio and demand comparative statics resulting from changes in $\bar{n}$ and $\bar{n}_{f}$ are equivalent in the following sense. Corresponding to changes in these supplies, the sign effect on the equilibrium price ratio depends on and only on the sign effect on the representative agent's demand. In the following result it will be useful to define income or wealth $I$ as

$$
I=p \bar{n}+p_{f} \bar{n}_{f} .
$$

Then changes in the endowments $\bar{n}$ or $\bar{n}_{f}$ can equivalently be viewed as changes in $I$ or, in terms of standard demand theory, income effects.

Proposition 1 Assume a single period, representative agent asset exchange economy, where the optimization problem is given by eqns. (2)-(3). Then ${ }^{6}$

$$
\left.\frac{\partial\left(\frac{p}{p_{f}}\right)}{\partial \bar{n}} \gtreqless 0 \Leftrightarrow \frac{\partial n_{f}}{\partial I}\right|_{n_{f}=\bar{n}_{f}} \lesseqgtr 0
$$

and

$$
\left.\frac{\partial\left(\frac{p}{p_{f}}\right)}{\partial \bar{n}_{f}} \gtreqless 0 \Leftrightarrow \frac{\partial n}{\partial I}\right|_{n=\bar{n}} \gtreqless 0 .
$$

Proof. Differentiating the first order condition eqn. (4) with respect to $\bar{n}$, we can obtain

$$
\frac{\partial}{\partial \bar{n}}\left(\frac{p}{p_{f}}\right)=\frac{W_{n, n} W_{n_{f}}-W_{n, n_{f}} W_{n}}{W_{n_{f}}^{2}} .
$$

Differentiating the first order condition with respect to the income $I$, we have

$$
W_{n, n} \frac{\partial n}{\partial I}+W_{n, n_{f}} \frac{\partial n_{f}}{\partial I}-\frac{W_{n}}{W_{n_{f}}}\left(W_{n, n_{f}} \frac{\partial n}{\partial I}+W_{n_{f}, n_{f}} \frac{\partial n_{f}}{\partial I}\right)=0 .
$$

\footnotetext{
${ }^{6}$ In an exchange economy since only relative prices matter, if one uses the normalization $p_{f}=1$ as in [9] and [21] then the comparative statics for $p$ will be the same as for $\frac{p}{p_{f}}$.
} 
Differentiating the budget constraint with respect to the income $I$, we have

$$
p \frac{\partial n}{\partial I}+p_{f} \frac{\partial n_{f}}{\partial I}=1
$$

Therefore, we have

$$
\left.\frac{\partial n_{f}}{\partial I}\right|_{n_{f}=\bar{n}_{f}}=-\frac{1}{p W_{n_{f}}} \frac{W_{n, n} W_{n_{f}}-W_{n, n_{f}} W_{n}}{2 W_{n, n_{f}}-\frac{p}{p_{f}} W_{n_{f}, n_{f}}-\frac{p_{f}}{p} W_{n, n}}
$$

Since $W\left(n, n_{f}\right)$ is concave, we always have

$$
W_{n, n} W_{n_{f}, n_{f}}-W_{n, n_{f}}^{2} \geq 0 \Leftrightarrow \sqrt{W_{n, n} W_{n_{f}, n_{f}}}-\left|W_{n, n_{f}}\right| \geq 0
$$

Then we have

$$
2 W_{n, n_{f}}-\frac{p}{p_{f}} W_{n_{f}, n_{f}}-\frac{p_{f}}{p} W_{n, n} \geq 2\left(\sqrt{W_{n, n} W_{n_{f}, n_{f}}}+W_{n, n_{f}}\right) \geq 2\left(\sqrt{W_{n, n} W_{n_{f}, n_{f}}}-\left|W_{n, n_{f}}\right|\right) \geq 0 .
$$

Therefore, we have

$$
\left.\frac{\partial\left(\frac{p}{p_{f}}\right)}{\partial \bar{n}} \gtreqless 0 \Leftrightarrow \frac{\partial n_{f}}{\partial I}\right|_{n_{f}=\bar{n}_{f}} \lesseqgtr 0
$$

Similarly, we can prove that

$$
\left.\frac{\partial\left(\frac{p}{p_{f}}\right)}{\partial \bar{n}_{f}} \gtreqless 0 \Leftrightarrow \frac{\partial n}{\partial I}\right|_{n=\bar{n}} \gtreqless 0 .
$$

Remark 1 It is important to note that the condition $\left.\frac{\partial n_{f}}{\partial I}\right|_{n_{f}=\bar{n}_{f}}>0$ does not correspond to the risk free asset being a normal good since $\bar{n}_{f}$ need not be positive. The derivatives of $n_{f}$ respect to I should be viewed as the slope of the risk free asset Engel curve. When goods can be negative, the appropriate definition of a normal good should be $n_{f} \frac{\partial n_{f}}{\partial I}>0$. Indeed there are four different sign combinations for the slope and whether the risk free asset is being held long or short. In a certainty setting, Nachbar [19] shows that if there are $L$ commodities and good $L$ is selected as the numeraire, then the price of good 1 will increase with its supply only if the composite commodity formed by the other L-1 commodities is an inferior good. Because for Nachbar commodities must be positive, the condition that the slope of a good's Engel curve is positive is equivalent to it being a normal good.

The geometric intuition for Proposition 1 can be expressed very simply in terms of Figure 2 . Let $\left(\bar{n}^{(0)}, \bar{n}_{f}\right)$ be the initial equilibrium point and denote the budget constraint passing through the point $L_{0}$. Suppose the corresponding income level is $I^{(0)}=p \bar{n}^{(0)}+p_{f} \bar{n}_{f}$. Now move to a new equilibrium point $\left(\bar{n}^{(1)}, \bar{n}_{f}\right)$ characterized by a larger endowment of the risky asset. Label the new constraint tangent to the indifference curve passing through $\left(\bar{n}^{(1)}, \bar{n}_{f}\right)$ as $L_{1}$. Using the approach in [11], the slope of the representative agent's indifference curve in the $n-n_{f}$ plane is given by

$$
\left.\frac{d n_{f}}{d n}\right|_{W=c o n s t}=-\frac{p}{p_{f}} .
$$

If $\frac{\partial\left(\frac{p}{p_{f}}\right)}{\partial \bar{n}}>0$ then $L_{1}$ will be steeper than $L_{0}$. Now consider another budget constraint $L_{2}$, which passes the point $\left(\bar{n}^{(1)}, \bar{n}_{f}\right)$ and parallel to $L_{0}$. Clearly $L_{2}$ corresponds to the same price vector as $L_{0}$, but the corresponding income level $I^{(1)}$ is larger than $I^{(0)}$. Since $L_{1}$ is also steeper than $L_{2}$, 


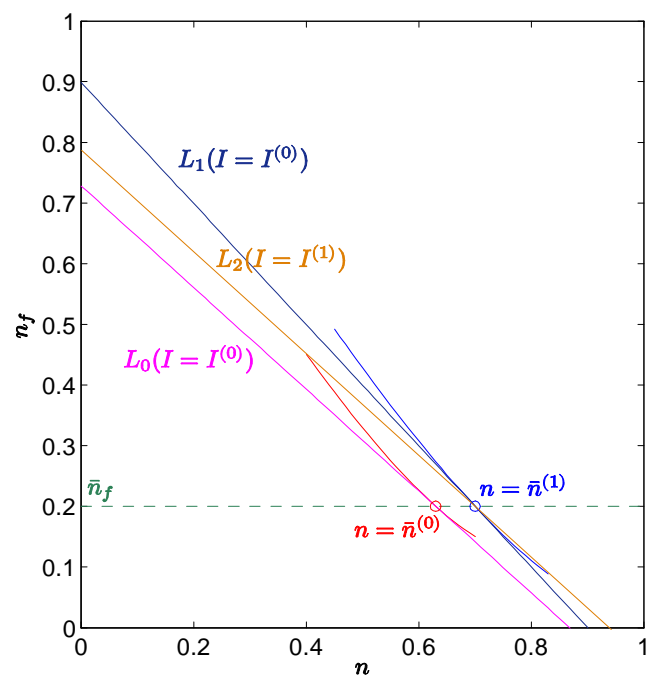

Figure 2:

the indifference curve tangent to $L_{1}$ will intersect with $L_{2}$ below $n_{f}=\bar{n}_{f}$. Due to the convexity of the indifference curve, the optimal point on $L_{2}$ is below the $n_{f}=\bar{n}_{f}$ line, implying $\left.\frac{\partial n_{f}}{\partial I}\right|_{n_{f}=\bar{n}_{f}}<0$. Therefore, we have the Proposition 1 conclusion. The case of increasing $\bar{n}_{f}$ can be discussed similarly.

Given Proposition 1, we can determine the comparative statics for the equilibrium price ratio once we specify the specific conditions determining the sign of the demand comparative statics. First defining the classic Arrow-Pratt [1]-[23] absolute and relative risk aversion measures corresponding to the representative agent's preferences as

$$
\tau_{A}={ }_{\text {def }}-\frac{W^{\prime \prime}\left(c_{2}\right)}{W^{\prime}\left(c_{2}\right)} \quad \text { and } \quad \tau_{R}={ }_{\text {def }}-c_{2} \frac{W^{\prime \prime}\left(c_{2}\right)}{W^{\prime}\left(c_{2}\right)},
$$

it follows from Arrow [1] that in a single risky asset and risk free asset setting, the risky asset will be a normal good, i.e. $\frac{\partial n}{\partial I}>0$, if (i) both assets are held long and (ii) $\tau_{A}^{\prime}<0$. If one additionally assumes (iii) $\tau_{R}^{\prime} \geq 0$, then Aura, Diamond, and Geanakoplos [2] point out that the risk free asset will also be a normal good, i.e. $\frac{\partial n_{f}}{\partial I}>0$. Therefore under (i)-(iii), we always have

$$
\frac{\partial}{\partial \bar{n}}\left(\frac{p\left(\bar{n}, \bar{n}_{f}\right)}{p_{f}\left(\bar{n}, \bar{n}_{f}\right)}\right)<0 \quad \text { and } \quad \frac{\partial}{\partial \bar{n}_{f}}\left(\frac{p\left(\bar{n}, \bar{n}_{f}\right)}{p_{f}\left(\bar{n}, \bar{n}_{f}\right)}\right)>0
$$

(See [14] for an in depth discussion of the signs of $\frac{\partial n}{\partial I}$ and $\frac{\partial n_{f}}{\partial I}$ when conditions (i) - (iii) do not hold in a complete market setting.)

The above discussion including Proposition 1 can be directly applied to the comparative statics results for the gross equity premium $Z$ which is defined as

$$
Z={ }_{\text {def }} \frac{E \widetilde{R}}{R_{f}}=\frac{p_{f}\left(\bar{n}, \bar{n}_{f}\right) E \widetilde{\xi}}{p\left(\bar{n}, \bar{n}_{f}\right) \xi_{f}} .
$$

Corollary 1 Assume a single period representative agent asset exchange economy, where the optimization problem is given by eqns. (2)-(3). If $\tau_{A}^{\prime} \leq 0$, then

$$
\left.\frac{\partial Z}{\partial \bar{n}} \gtreqless 0 \Leftrightarrow \frac{\partial n_{f}}{\partial I}\right|_{n_{f}=\bar{n}_{f}} \gtreqless 0
$$


and

$$
\frac{\partial Z}{\partial \bar{n}_{f}} \leq 0
$$

Proof. This follows directly from the definition of $Z$ and Proposition 1.

The seeming asymmetry of the $\frac{\partial Z}{\partial \bar{n}_{f}}$ result is a direct consequence of $\tau_{A}^{\prime} \leq 0$ implying that the risky asset is a normal good. It follows from Kubler, Selden and Wei [14] that if one allows either $n_{f}<0$ or $\tau_{R}^{\prime}<0$, then the Engel curve for the risk free asset can be downward sloping and it follows from Corollary 1 that increasing the supply of the risky asset $\bar{n}$ can result in a decrease in the gross equity premium. It will be noted that the comparative statics with respect to asset supplies for $Z$ and $\frac{p}{p_{f}}$ always change in an opposite direction. Throughout this paper, we will focus primarily on the comparative statics of $\frac{p}{p_{f}}$.

\subsection{Simple Preference Restrictions}

Given that the necessary and sufficient condition for the sign effects of changing the supply of the risky asset depends on whether risk free asset Engel curve is increasing or decreasing in income, it is natural to ask when this occurs given explicit restrictions on the representative agent's preferences. In principle one can use Kubler, Selden and Wei [14] to settle this question. However, we choose a different approach to prove the results since the proofs here will then extend directly to the two-period setting in the next section. The following proposition generalizes the above corollary.

Proposition 2 Assume a single period representative agent asset exchange economy, where the optimization problem is given by eqns. (2)-(3). Then we have

$$
\frac{\partial\left(\frac{p}{p_{f}}\right)}{\partial \bar{n}_{f}} \gtreqless 0 \Leftrightarrow \tau_{A}^{\prime} \lesseqgtr 0 .
$$

If $\tau_{A}^{\prime} \geq 0$, the we have

$$
\frac{\partial\left(\frac{p}{p_{f}}\right)}{\partial \bar{n}}<0
$$

If $\tau_{A}^{\prime}<0$

(i) $\tau_{R}^{\prime} \geq 0$ and $\bar{n}_{f} \geq 0$, then we have

$$
\frac{\partial\left(\frac{p}{p_{f}}\right)}{\partial \bar{n}} \leq 0
$$

(ii) $\tau_{R}^{\prime} \leq 0$ and $\bar{n}_{f} \leq 0$, then we have

$$
\frac{\partial\left(\frac{p}{p_{f}}\right)}{\partial \bar{n}} \geq 0
$$

where for both (i) and (ii) the equal sign can be reached if and only if $\bar{n}_{f}=0$ and $\tau_{R}^{\prime}=0$.

Proof. Differentiating the first order condition eqn. (4) with respect to $\bar{n}$ and noticing that

$$
E\left[\left(\widetilde{\xi}-\frac{p}{p_{f}} \xi_{f}\right) W^{\prime}\right]=0
$$

we can obtain

$$
\frac{\partial\left(\frac{p}{p_{f}}\right)}{\partial \bar{n}_{f}}=\frac{E W^{\prime} E\left[\left(\widetilde{\xi}-\frac{p}{p_{f}} \xi_{f}\right) W^{\prime \prime}\right]}{\left(E\left[W^{\prime}\left(\widetilde{\xi} \bar{n}+\xi_{f} \bar{n}_{f}\right)\right]\right)^{2}} .
$$


It follows from the generalized Chebyshev's Algebraic Inequality (see [8], Proposition 15 (2)) that if $\tau_{A}^{\prime} \lesseqgtr 0$, or equivalently, $\left(\frac{W^{\prime \prime}}{W^{\prime}}\right)^{\prime} \gtreqless 0$, then

$$
E\left[\left(\widetilde{\xi}-\frac{p}{p_{f}} \xi_{f}\right) W^{\prime}\left(\frac{W^{\prime \prime}}{W^{\prime}}\right)\right] \gtreqless E\left[\left(\widetilde{\xi}-\frac{p}{p_{f}} \xi_{f}\right) W^{\prime}\right] E\left[\frac{W^{\prime \prime}}{W^{\prime}}\right]=0 .
$$

Therefore, we have

$$
\frac{\partial\left(\frac{p}{p_{f}}\right)}{\partial \bar{n}_{f}} \gtreqless 0 \Leftrightarrow \tau_{A}^{\prime} \lesseqgtr 0 .
$$

Moreover, we have

$$
\frac{\partial\left(\frac{p}{p_{f}}\right)}{\partial \bar{n}}=\frac{E W^{\prime} E\left[\widetilde{\xi}\left(\widetilde{\xi}-\frac{p}{p_{f}} \xi_{f}\right) W^{\prime \prime}\right]}{\xi_{f}\left(E\left[W^{\prime}\left(\widetilde{\xi} \bar{n}+\xi_{f} \bar{n}_{f}\right)\right]\right)^{2}}=-\frac{E W^{\prime} E\left[\left(\widetilde{\xi}-\frac{p}{p_{f}} \xi_{f}\right) W^{\prime}\left(-\frac{\tilde{\xi} W^{\prime \prime}}{W^{\prime}}\right)\right]}{\xi_{f}\left(E\left[W^{\prime}\left(\widetilde{\xi} \bar{n}+\xi_{f} \bar{n}_{f}\right)\right]\right)^{2}} .
$$

If $\tau_{A}^{\prime} \geq 0$, then $-\frac{\widetilde{\xi} W^{\prime \prime}}{W^{\prime}}$ is a strictly increasing function of $\widetilde{\xi}$ and hence we have

$$
E\left[\left(\widetilde{\xi}-\frac{p}{p_{f}} \xi_{f}\right) W^{\prime}\left(-\frac{\widetilde{\xi} W^{\prime \prime}}{W^{\prime}}\right)\right]>E\left[\left(\widetilde{\xi}-\frac{p}{p_{f}} \xi_{f}\right) W^{\prime}\right] E\left[-\frac{\widetilde{\xi} W^{\prime \prime}}{W^{\prime}}\right]=0,
$$

which implies that

$$
\frac{\partial\left(\frac{p}{p_{f}}\right)}{\partial \bar{n}}<0
$$

When $\tau_{A}^{\prime}<0$, defining $\widetilde{c}_{2}=\widetilde{\xi} \bar{n}+\xi_{f} \bar{n}_{f}$, we have

$$
E\left[\widetilde{\xi}\left(\widetilde{\xi}-\frac{p}{p_{f}} \xi_{f}\right) W^{\prime \prime}\right]=E\left[\left(\widetilde{\xi}-\frac{p}{p_{f}} \xi_{f}\right) W^{\prime} \frac{\widetilde{c}_{2} W^{\prime \prime}}{W^{\prime}}\right]-\xi_{f} \bar{n}_{f} E\left[\left(\widetilde{\xi}-\frac{p}{p_{f}} \xi_{f}\right) W^{\prime \prime}\right] .
$$

Therefore, if $\tau_{R}^{\prime} \geq 0$ and $\bar{n}_{f} \geq 0$, then we have

$$
\frac{\partial\left(\frac{p}{p_{f}}\right)}{\partial \bar{n}} \leq 0
$$

and if $\tau_{R}^{\prime} \leq 0$ and $\bar{n}_{f} \leq 0$, then we have

$$
\frac{\partial\left(\frac{p}{p_{f}}\right)}{\partial \bar{n}} \geq 0
$$

where the equal sign can be reached if and only if $\bar{n}_{f}=0$ and $\tau_{R}^{\prime}=0$.

Remark 2 We have argued that under the assumption $\frac{E \widetilde{\xi}}{p}>\frac{\xi_{f}}{p_{f}}$, we have $n>0$. Since the sign of $\tau_{A}^{\prime}$ will fully determine whether the risky asset is a normal good or not, we have

$$
\tau_{A}^{\prime} \lesseqgtr 0 \Leftrightarrow \frac{\partial n}{\partial I} \gtreqless 0 \Leftrightarrow \frac{\partial\left(\frac{p}{p_{f}}\right)}{\partial \bar{n}_{f}} \gtreqless 0 .
$$

Moreover, if $\tau_{A}^{\prime} \leq 0$ and $\tau_{R}^{\prime} \geq 0$, then the risk free asset is a normal good when $n_{f}>0$, implying $\frac{\partial n_{f}}{\partial I}>0$ and $\frac{\partial\left(\frac{p}{p_{f}}\right)}{\partial \bar{n}}<0$. Similarly, if $\tau_{A}^{\prime} \leq 0$ and $\tau_{R}^{\prime} \leq 0$, then the risk free asset is a normal good when $n_{f}<0$, implying $\frac{\partial n_{f}}{\partial I}<0$ and $\frac{\partial\left(\frac{p}{p_{f}}\right)}{\partial \bar{n}}>0$. 
While Proposition 2 provides sufficient conditions for the cases where $\tau_{R}^{\prime} \bar{n}_{f} \geq 0$, it is silent on the cases where $\tau_{R}^{\prime} \bar{n}_{f} \leq 0$. However for the widely used HARA class, we are able to provide necessary and sufficient condition for each member.

Proposition 3 Assume the representative agent's preferences satisfy the standard expected utility axioms and the NM index $W\left(n, n_{f}\right)$ corresponds to the HARA class and that (5) and (6) are satisfied. Then

(i) If

$$
W\left(c_{2}\right)=-\frac{c_{2}^{-\delta}}{\delta}, \quad \delta>-1
$$

then

$$
\frac{\partial\left(\frac{p}{p_{f}}\right)}{\partial \bar{n}} \gtreqless 0 \Leftrightarrow \bar{n}_{f} \lesseqgtr 0,
$$

(ii) if

$$
W\left(c_{2}\right)=-\frac{\left(c_{2}-a\right)^{-\delta}}{\delta}, \quad \delta>-1, a>0
$$

then

$$
\frac{\partial\left(\frac{p}{p_{f}}\right)}{\partial \bar{n}} \gtreqless 0 \Leftrightarrow \bar{n}_{f} \lesseqgtr \frac{a}{\xi_{f}},
$$

(iii) If

$$
W\left(c_{2}\right)=-\frac{\left(c_{2}+a\right)^{-\delta}}{\delta}, \quad \delta>-1, a>0
$$

then

$$
\frac{\partial\left(\frac{p}{p_{f}}\right)}{\partial \bar{n}} \gtreqless 0 \Leftrightarrow \bar{n}_{f} \lesseqgtr-\frac{a}{\xi_{f}},
$$

(iv) If

$$
W\left(c_{2}\right)=-\frac{\exp \left(-\lambda c_{2}\right)}{\lambda}, \quad \lambda>0
$$

then

$$
\frac{\partial\left(\frac{p}{p_{f}}\right)}{\partial \bar{n}}<0
$$

(v) If

$$
W\left(c_{2}\right)=q c_{2}-c_{2}^{2}, \quad q>0
$$

then

$$
\frac{\partial\left(\frac{p}{p_{f}}\right)}{\partial \bar{n}}<0 .
$$

Proof. We apply a similar method as in the proof of Proposition 2, which does rely on the corresponding demand properties. For case (i), we have

$$
\frac{p}{p_{f}}=\frac{E\left[\widetilde{\xi}\left(\widetilde{\xi} \bar{n}+\xi_{f} \bar{n}_{f}\right)^{-1-\delta}\right]}{E\left[\xi_{f}\left(\widetilde{\xi} \bar{n}+\xi_{f} \bar{n}_{f}\right)^{-1-\delta}\right]}
$$


Therefore,

$$
\frac{\partial\left(\frac{p}{p_{f}}\right)}{\partial \bar{n}}=\frac{(1+\delta) \xi_{f} A}{\left(E\left[\xi_{f}\left(\widetilde{\xi} \bar{n}+\xi_{f} \bar{n}_{f}\right)^{-1-\delta}\right]\right)^{2}},
$$

where

$$
A=E\left[\widetilde{\xi}\left(\widetilde{\xi} \bar{n}+\xi_{f} \bar{n}_{f}\right)^{-2-\delta}\right] E\left[\widetilde{\xi}\left(\widetilde{\xi} \bar{n}+\xi_{f} \bar{n}_{f}\right)^{-1-\delta}\right]-E\left[\widetilde{\xi}^{2}\left(\widetilde{\xi} \bar{n}+\xi_{f} \bar{n}_{f}\right)^{-2-\delta}\right] E\left[\left(\widetilde{\xi} \bar{n}+\xi_{f} \bar{n}_{f}\right)^{-1-\delta}\right] .
$$

After some algebra, we can rewrite $A$ as

$$
\begin{aligned}
A= & \bar{n}_{f} E\left[\widetilde{\xi}\left(\widetilde{\xi} \bar{n}+\xi_{f} \bar{n}_{f}\right)^{-2-\delta}\right] E\left[\xi_{f}\left(\widetilde{\xi} \bar{n}+\xi_{f} \bar{n}_{f}\right)^{-1-\delta}\right] \\
& -\bar{n}_{f} E\left[\xi_{f}\left(\widetilde{\xi} \bar{n}+\xi_{f} \bar{n}_{f}\right)^{-2-\delta}\right] E\left[\widetilde{\xi}\left(\widetilde{\xi} \bar{n}+\xi_{f} \bar{n}_{f}\right)^{-1-\delta}\right] .
\end{aligned}
$$

Noticing that

$$
E\left[\widetilde{\xi}\left(\widetilde{\xi} \bar{n}+\xi_{f} \bar{n}_{f}\right)^{-1-\delta}\right]=\frac{p}{p_{f}} E\left[\xi_{f}\left(\widetilde{\xi} \bar{n}+\xi_{f} \bar{n}_{f}\right)^{-1-\delta}\right]
$$

we can rewrite $A$ as

$$
A=\bar{n}_{f} E\left[\xi_{f}\left(\widetilde{\xi} \bar{n}+\xi_{f} \bar{n}_{f}\right)^{-1-\delta}\right] E\left[\left(\widetilde{\xi}-\frac{p}{p_{f}} \xi_{f}\right)\left(\widetilde{\xi} \bar{n}+\xi_{f} \bar{n}_{f}\right)^{-2-\delta}\right] .
$$

It follows from a non-monotonic version of Chebyshev's Algebraic Inequality that

$$
E\left[\left(\widetilde{\xi}-\frac{p}{p_{f}} \xi_{f}\right)\left(\widetilde{\xi} \bar{n}+\xi_{f} \bar{n}_{f}\right)^{-2-\delta}\right]<0 .
$$

Therefore, we can conclude that

$$
\bar{n}_{f} \gtreqless 0 \Leftrightarrow A \lesseqgtr 0 \Leftrightarrow \frac{\partial\left(\frac{p}{p_{f}}\right)}{\partial \bar{n}} \lesseqgtr 0 .
$$

For case (ii), defining

$$
\bar{n}_{f}^{\text {new }}=\bar{n}_{f}-\frac{a}{\xi_{f}},
$$

it follows from the the same steps as above that

$$
\bar{n}_{f}^{\text {new }} \gtreqless 0 \Leftrightarrow \bar{n}_{f} \lesseqgtr \frac{a}{\xi_{f}} \Leftrightarrow \frac{\partial\left(\frac{p}{p_{f}}\right)}{\partial \bar{n}} \lesseqgtr 0 .
$$

For case (iii), defining

$$
\bar{n}_{f}^{\text {new }}=\bar{n}_{f}+\frac{a}{\xi_{f}},
$$

it follows from the the same steps as above that

$$
\bar{n}_{f}^{\text {new }} \gtreqless 0 \Leftrightarrow \bar{n}_{f} \lesseqgtr-\frac{a}{\xi_{f}} \Leftrightarrow \frac{\partial\left(\frac{p}{p_{f}}\right)}{\partial \bar{n}} \lesseqgtr 0 .
$$

Case (iv) and (v) have been proved in Proposition 2.

Although the proof of Proposition 3, does not rely at all on the demand properties of the HARA utility functions, the following Example provides intuition into the connection to the Engel curve properties given in Proposition 1. 


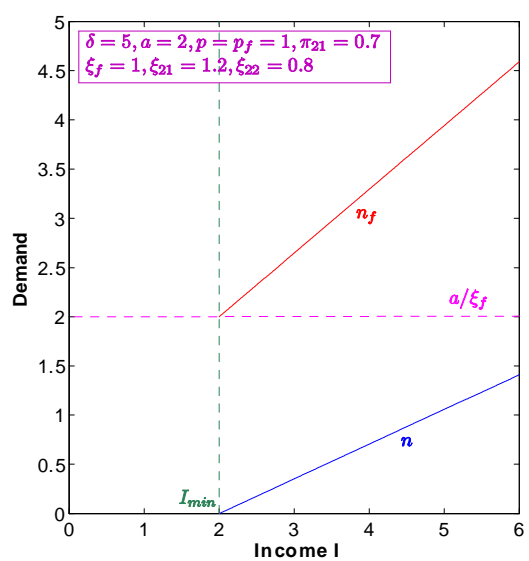

(a)

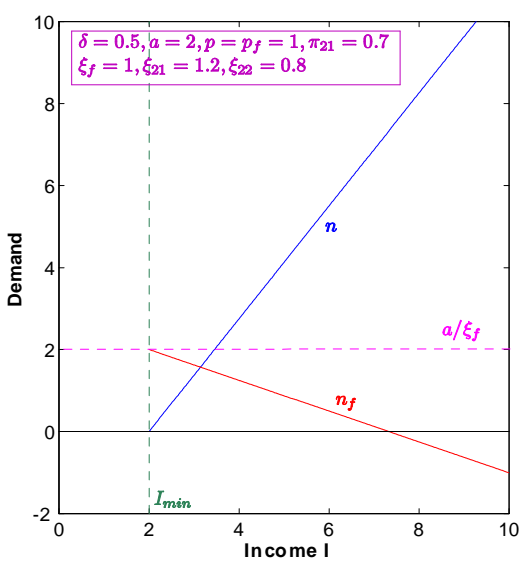

(b)

Figure 3:

Example 1 Assume the representative agent's preferences are represented by the HARA utility

$$
W\left(c_{2}\right)=-\frac{\left(c_{2}-a\right)^{-\delta}}{\delta}
$$

where $a>0$ and $\delta>-1$. For simplicity, let the random variable $\widetilde{\xi}$ take the values $\xi_{21}$ with probability $\pi_{21}$ and $\xi_{22}$ with the probability $\pi_{22}=1-\pi_{21}$. The corresponding Engel curves for two different sets of parameters are plotted in Figure 3. It is clear that

$$
\frac{\partial n_{f}}{\partial I} \gtrless 0 \Leftrightarrow n_{f} \gtrless \frac{a}{\xi_{f}} .
$$

Therefore in equilibrium, the comparative statics of $\frac{p}{p_{f}}$ corresponding to changes in the supply of the risky asset, $\bar{n}$, are completely determined by a comparison between $\bar{n}_{f}$ and $\frac{a}{\xi_{f}}$.

The following illustrates the important case where asset Engel curves are non-linear and hence not covered by Proposition 3 .

Example 2 Assume the representative agent's preferences satisfy the standard expected utility axioms and the NM index is given by

$$
W\left(c_{2}\right)=-\frac{c_{2}^{-\delta_{1}}}{\delta_{1}}-\frac{c_{2}^{-\delta_{2}}}{\delta_{2}}, \quad \delta_{1}>\delta_{2} \geq-1 .
$$

It can be verified that $\tau_{A}^{\prime}<0$ and $\tau_{R}^{\prime}<0$. Therefore, if $\bar{n}_{f}<0$, we have

$$
\frac{\partial\left(\frac{p}{p_{f}}\right)}{\partial \bar{n}}>0 .
$$

Next we consider the case when $\bar{n}_{f}>0$. Assume that

$$
\widetilde{\xi}=\left\{\begin{array}{ll}
\xi_{21} & \left(\pi=\pi_{21}\right) \\
\xi_{22} & \left(\pi=\pi_{22}\right)
\end{array} .\right.
$$




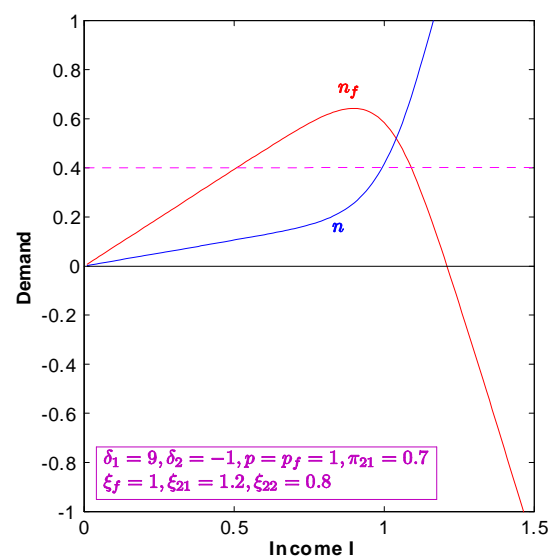

(a)

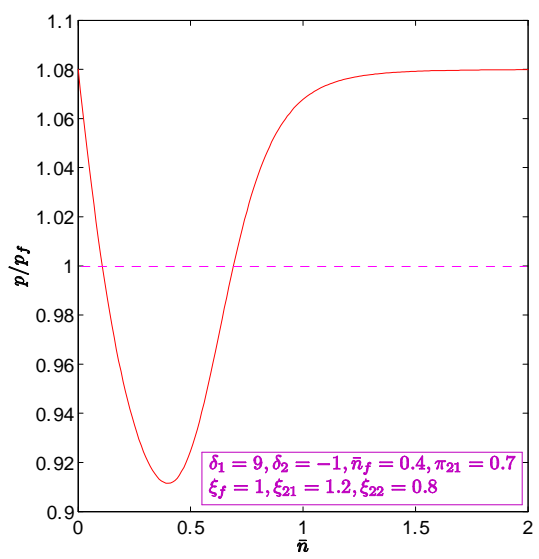

(b)

Figure 4:

The first order condition gives that

$$
\frac{p}{p_{f}}=\frac{\sum_{i=1}^{2} \pi_{2 i} \xi_{2 i}\left(\left(\xi_{2 i} \bar{n}+\xi_{f} \bar{n}_{f}\right)^{-1-\delta_{1}}+\left(\xi_{2 i} \bar{n}+\xi_{f} \bar{n}_{f}\right)^{-1-\delta_{2}}\right)}{\sum_{i=1}^{2} \pi_{2 i} \xi_{f}\left(\left(\xi_{2 i} \bar{n}+\xi_{f} \bar{n}_{f}\right)^{-1-\delta_{1}}+\left(\xi_{2 i} \bar{n}+\xi_{f} \bar{n}_{f}\right)^{-1-\delta_{2}}\right)} .
$$

We plot the Engel curves in Figure $4(a)$ and $\frac{p}{p_{f}}$ versus $\bar{n}$ in Figure $4(b)$. From Figure $4(b)$, clearly $\frac{p}{p_{f}}$ is not monotone in $\bar{n}$. The intuition can be understood as follows. In Figure 4(a), we consider the line $n_{f}=0.4$ and there are two income levels that correspond to this level of risk free asset demand. At the lower income level, we have $\frac{\partial n_{f}}{\partial I}>0$ and at the higher income level, we have $\frac{\partial n_{f}}{\partial I}<0 . \quad$ Therefore, if we choose $\bar{n}_{f}=0.4$ in equilibrium, we need also specify the value of $\bar{n}$ to determine which equilibrium we are considering. This is very different from the HARA class discussed in Proposition 3 since the Engel curves are linear there. In Figure 4(b), we draw a line corresponding to $\frac{p}{p_{f}}=1$. The $\bar{n}$ values corresponding to the intersection points between the line and the $\frac{p}{p_{f}}$ curves are exactly the two $\bar{n}$ values we need specify for the equilibrium. For the smaller $\bar{n}$ value, we are at the low income level equilibrium in Figure $4(a)$ and hence $\frac{\partial n_{f}}{\partial I}>0$, which is consistent with the behavior that $\frac{\partial\left(\frac{p}{p_{f}}\right)}{\partial \bar{n}}<0$ in Figure $4(b)$. For the larger $\bar{n}$ is value, we are at the high income level equilibrium in Figure $4(a)$ and hence $\frac{\partial n_{f}}{\partial I}<0$, which is consistent with the behavior that $\frac{\partial\left(\frac{p}{p_{f}}\right)}{\partial \bar{n}}>0$ in Figure $4(b)$.

\section{Two Period Comparative Statics}

In the two period setting, the equivalence between the single period demand and equilibrium price ratio comparative statics breaks down. While the former becomes more complex, fortunately the latter which is our primary interest tends not to. 


\subsection{Economy}

The representative agent's two period preferences are defined over certain period one and random period two consumption pairs $\left(c_{1}, \widetilde{c}_{2}\right)$ and satisfy the appropriate expected utility axioms. The NM index $W\left(c_{1}, c_{2}\right)$ is additively separable and $E W\left(c_{1}, \widetilde{c}_{2}\right)$ is given by

$$
W\left(c_{1}, n, n_{f}\right)=W_{1}\left(c_{1}\right)+E W_{2}\left(\widetilde{\xi} n+\xi_{f} n_{f}\right) .
$$

The period two index $W_{2}$ exhibits the same properties as the single period NM utility, $W_{2} \in C^{3}$, $W_{2}^{\prime}>0, W_{2}^{\prime \prime} \leq 0$. The function $W_{1}$ satisfies $W_{1} \in C^{2}, W_{1}^{\prime}>0, W_{1}^{\prime \prime}<0$. Finally, we assume the asset return properties introduced in Section 2 continue to hold.

The optimization problem of the representative agent becomes

$$
\max _{c_{1}, n, n_{f}} W\left(c_{1}, n, n_{f}\right)=\max _{c_{1}, n, n_{f}}\left(W_{1}\left(c_{1}\right)+E W_{2}\left(\widetilde{\xi} n+\xi_{f} n_{f}\right)\right),
$$

subject to the exchange economy constraint

$$
p_{1} \bar{c}_{1}+p \bar{n}+p_{f} \bar{n}_{f}=p_{1} c_{1}+p n+p_{f} n_{f}
$$

where $p_{1}$ and $\bar{c}_{1}>0$ denote respectively the price and endowment of period one consumption. The resulting first order conditions are given by

$$
\begin{gathered}
\frac{W_{n}}{W_{c_{1}}}=\frac{\left[\widetilde{\xi} W_{2}^{\prime}\left(\widetilde{\xi} n+\xi_{f} n_{f}\right)\right]}{W_{1}^{\prime}\left(c_{1}\right)}=\frac{p}{p_{1}}, \\
\frac{W_{n_{f}}}{W_{c_{1}}}=\frac{E\left[\xi_{f} W_{2}^{\prime}\left(\widetilde{\xi} n+\xi_{f} n_{f}\right)\right]}{W_{1}^{\prime}\left(c_{1}\right)}=\frac{p_{f}}{p_{1}}, \\
\frac{W_{n}}{W_{n_{f}}}=\frac{E\left[\widetilde{\xi} W_{2}^{\prime}\left(\widetilde{\xi} n+\xi_{f} n_{f}\right)\right]}{E\left[\xi_{f} W_{2}^{\prime}\left(\widetilde{\xi} n+\xi_{f} n_{f}\right)\right]}=\frac{p}{p_{f}} .
\end{gathered}
$$

Since eqn. (72) is identical to the single period first order condition eqn. (4) assuming $W_{2}$ is affinely equivalent to the one period NM index, it follows immediately that the equilibrium price ratio $\frac{p}{p_{f}}$ and gross equity premium $Z$ remain the same as in the single period setting. Thus Propositions 2 and 3 extend without change. It should be noted that the fact that Proposition 1 may change even when $W$ is additively separable is of no practical significance given that Propositions 2 and 3 remain the same. ${ }^{7}$

\subsection{Equilibrium Return and Equity Premium Behavior}

By moving to two periods, we are able to derive comparative statics results for the equilibrium risk free return and the risky asset expected return. ${ }^{8}$

\footnotetext{
${ }^{7}$ It can be shown that if the composite commodity $p n+p_{f} n_{f}$ is a normal good, then Proposition 1 can be extended to the two period case. (See Nachbar [19] for a different application of the composite commodity in considering when the price of a good increases with its supply.) However, testing whether the composite commodity is a normal good may not be easy in practice. But, if one assumes complete markets then $p n+p_{f} n_{f}+=p_{21} c_{21}+p_{22} c_{22}$ and this is always a normal good.

${ }^{8}$ It is straightforward to show that if the representative agent's optimization is given by eqns. (68) - (69), then following the proof of Proposition 4, we have

$$
\frac{\partial E \widetilde{R}}{\partial \bar{c}_{1}}<0 \quad \text { and } \quad \frac{\partial R_{f}}{\partial \bar{c}_{1}}<0
$$
}


Proposition 4 Assume a two period representative agent asset exchange economy, where the optimization problem is given by eqns. (68) - (69) and W satisfies the conditions specified. Taking first period consumption as the numeraire, we have

$$
\frac{\partial R_{f}}{\partial \bar{n}}>0 \quad \text { and } \quad \frac{\partial R_{f}}{\partial \bar{n}_{f}}>0
$$

and

$$
\frac{\partial E \widetilde{R}}{\partial \bar{n}}>0 \quad \text { and } \quad \frac{\partial E \widetilde{R}}{\partial \bar{n}_{f}}>0 .
$$

Proof. Using the normalization $p_{1}=1$, it follows directly from the first order conditions eqns. (70)-(71) that

$$
R_{f}=\frac{\xi_{f}}{p_{f}}=\frac{W_{1}^{\prime}\left(\bar{c}_{1}\right)}{E W_{2}^{\prime}\left(\widetilde{\xi} \bar{n}+\xi_{f} \bar{n}_{f}\right)}
$$

and

$$
E \widetilde{R}=\frac{E \widetilde{\xi}}{p}=\frac{W_{1}^{\prime}\left(\bar{c}_{1}\right) E \widetilde{\xi}}{E\left[\widetilde{\xi} W_{2}^{\prime}\left(\widetilde{\xi} \bar{n}+\xi_{f} \bar{n}_{f}\right)\right]} .
$$

Since we assume that $\xi_{f}, \widetilde{\xi}>0$, we have

$$
\frac{\partial E W_{2}^{\prime}}{\partial \bar{n}}=E\left[\widetilde{\xi} W_{2}^{\prime \prime}\right]<0 \text { and } \frac{\partial E W_{2}^{\prime}}{\partial \bar{n}_{f}}=E\left[\xi_{f} W_{2}^{\prime \prime}\right]<0
$$

and

$$
\frac{\partial E\left[\widetilde{\xi} W_{2}^{\prime}\right]}{\partial \bar{n}}=E\left[\widetilde{\xi}^{2} W_{2}^{\prime \prime}\right]<0 \text { and } \frac{\partial E\left[\widetilde{\xi} W_{2}^{\prime}\right]}{\partial \bar{n}_{f}}=E\left[\xi_{f} \widetilde{\xi} W_{2}^{\prime \prime}\right]<0 .
$$

Therefore, we have

$$
\frac{\partial R_{f}}{\partial \bar{n}}>0 \quad \text { and } \quad \frac{\partial R_{f}}{\partial \bar{n}_{f}}>0
$$

and

$$
\frac{\partial E \widetilde{R}}{\partial \bar{n}}>0 \quad \text { and } \quad \frac{\partial E \widetilde{R}}{\partial \bar{n}_{f}}>0
$$

One may wonder why the equilibrium return comparative statics do not require restrictions paralleling those for $Z$. It follows from the the concavity of $W_{2}$ that an increase in any asset's supply will increase the relative abundance of period two consumption versus period one consumption. The marginal utility of period two consumption will decrease while that for period one will remain unchanged causing the price of either asset to decline. Since the payoff on the asset does not change, it follows from eqns. (70) and (71) that for each asset its return will increase.

\subsection{Non Additively Separable Expected Utility}

We consider the habit persistence generalization of additively separable NM utility (67) which was originally introduced to reconcile the equity premium puzzle (see Constantinides [6]). ${ }^{9}$ Following and

$$
\frac{\partial Z}{\partial \bar{c}_{1}}=0
$$

${ }^{9}$ See $[25]$ for a summary of the applications of this form of utility to asset pricing, macro, monetary policy and business-cycle theory. 
the classic certainty literature, the NM utility (67) is typically modified as follows resulting in $W$ no longer being additively separable

$$
W\left(c_{1}, n, n_{f}\right)=W_{1}\left(c_{1}\right)+E W_{2}\left(\widetilde{\xi} n+\xi_{f} n_{f}-\alpha c_{1}\right),
$$

where $\alpha>0$ and $\frac{\partial W}{\partial c_{1}}>0$. The standard interpretation for the habit persistence term $-\alpha c_{1}$ follows from the simple certainty setting. Assuming $W$ is a function of current and future consumption, the corresponding intuition for habit persistence is that "the more I eat in period one the hungrier I get in period two" [25].

Continuing to maintain the assumptions made for the two period additively separable $W$, the optimization problem of the representative agent becomes

$$
\max _{c_{1}, n, n_{f}} W\left(c_{1}, n, n_{f}\right)=\max _{c_{1}, n, n_{f}}\left(W_{1}\left(c_{1}\right)+E W_{2}\left(\widetilde{\xi} n+\xi_{f} n_{f}-\alpha c_{1}\right)\right)
$$

subject to the exchange economy constraint

$$
p_{1} c_{1}+p n+p_{f} n_{f}=p_{1} \bar{c}_{1}+p \bar{n}+p_{f} \bar{n}_{f}
$$

The resulting first order conditions are given by

$$
\begin{aligned}
\frac{W_{n_{f}}}{W_{c_{1}}} & =\frac{\xi_{f} E W_{2}^{\prime}}{W_{1}^{\prime}\left(\bar{c}_{1}\right)-\alpha E W_{2}^{\prime}}=\frac{p_{f}}{p_{1}}, \\
\frac{W_{n}}{W_{c_{1}}} & =\frac{E \tilde{\xi} W_{2}^{\prime}}{W_{1}^{\prime}\left(\bar{c}_{1}\right)-\alpha E W_{2}^{\prime}}=\frac{p}{p_{1}}
\end{aligned}
$$

and

$$
\frac{W_{n}}{W_{n_{f}}}=\frac{E\left[\widetilde{\xi} W_{2}^{\prime}\left(\widetilde{\xi} n+\xi_{f} n_{f}-\alpha c_{1}\right)\right]}{E\left[\xi_{f} W_{2}^{\prime}\left(\widetilde{\xi} n+\xi_{f} n_{f}-\alpha c_{1}\right)\right]}=\frac{p}{p_{f}} .
$$

Since at equilibrium, $-\alpha c_{1}$ is a constant in eqn. (86), if we define

$$
\bar{n}_{f}^{\text {new }}=\bar{n}_{f}-\frac{\alpha \bar{c}_{1}}{\xi_{f}}
$$

Propositions 2 and 3 extend to the current setting. This can be seen in the case of the latter from the modification of conditions (i)-(iii) given below.

Corollary 2 Assume a two period representative agent asset exchange economy, where the optimization problem is given by eqns. (82) - (83). Further assume the representative agent's preferences satisfy the standard expected utility axioms and the $N M$ index $W_{2}\left(c_{2}-\alpha c_{1}\right)$ corresponds to the HARA class and that (5) and (6) are satisfied. If

$$
W_{2}\left(c_{2}-\alpha c_{1}\right)=-\frac{\left(c_{2}-\alpha c_{1}-a\right)^{-\delta}}{\delta}, \quad \delta>-1, a \gtreqless 0,
$$

then

$$
\frac{\partial\left(\frac{p}{p_{f}}\right)}{\partial \bar{n}} \gtreqless 0 \Leftrightarrow \bar{n}_{f} \lesseqgtr \frac{a+\alpha \bar{c}_{1}}{\xi_{f}} .
$$

Proof. The proof directly follows from the proof of Proposition 3.

It is interesting to note that increasing $\bar{c}_{1}$ increases the range of risk-free asset supply over which the equity premium decreases with supply. Also note that if $a<0$, the comparative statics result will depend on the relative size of $|a|$ and $\alpha \bar{c}_{1}$. 


\section{Concluding Comments}

In this paper, we investigate the comparative statics of equilibrium prices, returns and the gross equity premium corresponding to changes in asset supplies in both single period and two period representative agent exchange economies. In the single period case, we demonstrate an equivalence between the comparative statics of asset demand and equilibrium prices. Several sufficient conditions are given for the response of equilibrium prices to changes in asset supplies. Also necessary and sufficient conditions are given for the popular HARA class of utilities. We show that surprisingly the price of the risky asset can increase with increasing supply for perfectly standard forms of expected utility. When considering the two period case, unfortunately the equivalence between the demand and equilibrium price comparative statics breaks down. However most of the equilibrium price and gross equity premium comparative statics carryover from the one period case. And if we make the perfectly natural choice of period one consumption as the numeraire, we can derive simple sufficient conditions for the comparative statics of equilibrium asset returns.

These results suggest two areas for future research. First, having based our analysis on the representative agent model, it would seem natural to consider similar comparative statics analyses for economies with heterogeneous agents. Second, given the results obtained in this paper and the recent appearance of a number of equilibrium models with a positive supply of risk free bonds, such as those discussed earlier, it would seem potentially fruitful to extend our analysis to Macro settings in which one endogenizes increases in the supply of bonds by a Government and equities by corporations.

\section{References}

[1] Arrow, K., Essays in the Theory of Risk Bearing, Markham, Chicago (1971).

[2] Aura, S., Diamond, P. and Geanakoplos, G., "Savings and Portfolio Choice in a Two-period Two-asset Model", American Economic Review 92 (2002), 1185-1191.

[3] Barsky, R., "Why Don't the Prices of Stocks and Bonds Move Together?", American Economic Review 79 (1989), 1132-1145.

[4] Cass, D. and Pavlova, A., "On Trees and Logs", Journal of Economic Theory 116 (2004), $41-83$.

[5] Cochrane, J., Longstaff, F. and Santa-Clara, P., "Two Trees", Review of Financial Studies 21 (2008), 347-385.

[6] Constantinides, G., "Habit Formation: A Resolution of the Equity Premium Puzzle", Journal of Political Economy 98 (1990), 519-543.

[7] Favilukis, J., S. Ludvigson and S. V. Nieuwerburgh, "The Macroeconomic Effects of Housing Wealth, Housing Finance, and Limited Risk-Sharing in General Equilibrium", SSRN 1602163 (2011).

[8] Gollier, C., The Economics of Risk and Time, MIT Press (2001).

[9] Gollier, C. and Schlesinger, H., "Changes in Risk and Asset Prices", Journal of Monetary Economics 49 (2002) 747-760. 
[10] Heaton, J. and Lucas, D., "Evaluating the Effects of Incomplete Markets on Risk Sharing and Asset Pricing", Journal of Political Economy 104 (1996), 443-487.

[11] Katzner, D., "A Simple Approach to Existence and Uniqueness of Competitive Equilibria", American Economic Review 62 (1972), 432-437.

[12] Katzner, D., "Static Demand Theory", MacMillan, New York (1970).

[13] Kohli, U., "Inverse Demand and Anti-Giffen Goods", European Economic Review 27 (1985), 397-404.

[14] Kubler, F., Selden, L. and Wei, X., "Inferior good and Giffen Behavior for Investing and Borrowing", working paper (2010).

[15] Lucas, R., "Asset Prices in an Exchange Economy", Econometrica 46 (1978), 1429-1445.

[16] Malinvaud, E., "Lectures on Microeconomic Theory", New York: North-Holland (1972).

[17] Mehra, R. and Prescott, E., "The Equity Premium: A Puzzle," Journal of Monetary Economics 15 (1985), 145-161.

[18] Meyer, D. and Meyer, J., "Risk Preferences in Multi-period Consumption Models, the Equity Premium Puzzle, and Habit Formation Utility", Journal of Monetary Economics 52 (2005), 1497-1515.

[19] Nachbar, J., "The last word on Giffen goods?", Economic Theory 11 (1998), 403-412.

[20] Nachbar, J., "General Equilibrium Comparative Statics", Econometrica 70 (2002), 2065-2074.

[21] Ohnishi, M. and Osaki, Y., "The Monotonicity of Asset Prices with Changes in Risk", Discussion Paper (2005).

[22] Parlour, C., Stanton, R. and Walden, J., "Revisiting Asset Pricing Anomalies in an Exchange Economy", Review of Financial Studies (forthcoming).

[23] Pratt, J., "Risk Aversion in the Small and in the Large", Econometrica 32 (1964), 122-136.

[24] Quah, J., "Market Demand and Comparative Statics When Goods Are Normal", Journal of Mathematical Economics 39 (2003), 317-333.

[25] Schmitt-Grohé, S. and Uribe, M., "Habit Persistence", The New Palgrave Dictionary of Economics, Second Edition. Eds. Steven N. Durlauf and Lawrence E. Blume. Palgrave Macmillan (2008).

[26] Weil, P., "The Equity Premium Puzzle and the Riskfree Rate Puzzle", J. Monetary Econ 24 (1989), 401-421. 